PROCEEDINGS OF THE

AMERICAN MATHEMATICAL SOCIETY

Volume 129, Number 12, Pages 3683-3690

S 0002-9939(01)06248-7

Article electronically published on June 27, 2001

\title{
THE SETS OF MONOMORPHISMS AND OF ALMOST OPEN OPERATORS BETWEEN LOCALLY CONVEX SPACES
}

\author{
JOSÉ BONET AND JOSÉ A. CONEJERO
}

(Communicated by Jonathan M. Borwein)

\begin{abstract}
If the set of monomorphisms between locally convex spaces is not empty, then it is an open subset of the space of all continuous and linear operators endowed with the topology of the uniform convergence on the bounded sets if and only if the domain space is normable. The corresponding characterization for the set of almost open operators is also obtained; it is related to the lifting of bounded sets and to the quasinormability of the domain space. Other properties and examples are analyzed.
\end{abstract}

\section{INTRODUCTION AND PRELIMINARIES}

Bounded below and almost open continuous linear operators between normed spaces, their relation with the topological divisors of zero in the normed algebra of all operators, and the approximate point spectrum have been extensively studied. We refer to the books of Berberian [3, Sections 56, 57], Harte [8], and the articles of Abramovich, Aliprantis and Polyrakis [2] and Harte [9]. In [2, Proposition 2.2] it is shown that the set of bounded below operators (or monomorphisms) between two normed spaces $X$ and $Y$ is open in the normed space of operators $L(X, Y)$. The corresponding result for almost open operators can be seen in [8, Theorem 3.4.3]. These results are extensions of the well-known fact (see e.g. [10, Theorem 18.12]) that the set of isomorphisms from a Banach space $X$ onto a Banach space $Y$ is an open subset of the Banach space $L(X, Y)$, a result which can be proved using the Neumann series for a linear operator. We refer to the recent article by Casazza and Kalton [5] for extensions of Paley-Wiener perturbation theory. In the case of continuous linear operators from a locally convex space $E$ into itself, it was proved by Kasahara in 1972 in [12] that if the set of isomorphisms is open in the space of operators $L_{b}(E)$ endowed with the topology of the uniform convergence on the bounded subsets of $E$, then the space $E$ must be normable (see the quotation in M. Akkar [1]). If $E$ is complete, in the terminology of topological algebras, $L_{b}(E)$ is a $Q$-algebra if and only if $E$ is normable.

Received by the editors May 2, 2000.

2000 Mathematics Subject Classification. Primary 46A32, 46A03, 46H35, 47A05, 47L05.

Key words and phrases. Bounded below operators, monomorphisms, almost open operators, locally convex spaces, quasinormable spaces.

The authors were partially supported by the project DGESIC, PB97-0333.

The second author was also supported by the Universidad Politécnica de Valencia, grant 19980998. 
The purpose of this note is to characterize completely when the set of monomorphisms and the set of almost open operators are open in the set of all operators $L(E, F)$ between two locally convex spaces $E$ and $F$ for the topology of uniform convergence on the bounded subsets of $E$. The characterizations are obtained in Theorems 2.1 and 2.2. The role of the quasinormability of the space $E$ is analyzed in Proposition 2.4 and Corollary 2.5. Theorem 2.6 studies the set of surjective and open operators between non-complete normed spaces. Several examples and propositions discuss other properties of these classes of operators.

Our notation for Banach spaces, operator theory and locally convex spaces is standard and we refer to the books [3, 8, 10, 11, 15, 17, 18. Unless explicitly mentioned, $E$ and $F$ denote Hausdorff locally convex spaces. If $E$ is a locally convex space, the set of continuous seminorms on $E$ is denoted by $\operatorname{cs}(E)$. If $p \in$ $\operatorname{cs}(E)$, the unit ball of this seminorm is denoted by $U_{p}:=\{x \in E \mid p(x) \leq 1\}$. The family of all closed absolutely convex neighbourhoods of the origin in $E$ is denoted by $\mathcal{U}_{0}(E)$. The Minkowski functional of $U \in \mathcal{U}_{0}(E)$ is denoted by $p_{U}$, and $p_{U} \in \operatorname{cs}(E)$. If $E$ is a normed space with the norm $\|$.$\| , its closed unit ball$ is denoted by $U_{E}:=\{x \in E \mid\|x\| \leq 1\}$. The set of all closed absolutely convex bounded subsets of a locally convex space $E$ is denoted by $\mathcal{B}(E)$. If $B \in \mathcal{B}(E)$, we denote by $E_{B}$ the normed space given by the linear span of $B$ endowed with the norm defined by the Minkowski functional $p_{B}$ of $B$. The set $B \in \mathcal{B}(E)$ is a Banach disc if $E_{B}$ is a Banach space. The space $E$ is called locally complete if every $B \in \mathcal{B}(E)$ is a Banach disc. Every complete space is locally complete.

As usual, the symbol $L_{b}(E, F)$ denotes the locally convex space of all (always linear and continuous) operators from a locally convex space $E$ into a locally convex space $F$, endowed with the locally convex topology of uniform convergence on the bounded subsets of $E$. A basis of neighbourhoods of zero of $L_{b}(E, F)$ is given by the sets

$$
\mathcal{W}(B, V):=\{T \in L(E, F) \mid T(B) \subseteq V\}
$$

as $B$ runs in $\mathcal{B}(E)$ and $V$ runs in $\mathcal{U}_{0}(F)$. This topology is defined by the seminorms $q_{B}(T):=\sup \{q(T(b)) \mid b \in B\}$, for $B \in \mathcal{B}(E)$ and $q \in c s(F)$. If $E$ and $F$ are normed, then $L_{b}(E, F)$ is the usual normed space of operators. If $E=F$, we write $L_{b}(E)$.

An operator $T \in L(E, F)$ is called bounded below if for every $p \in c s(E)$ there is $q \in \operatorname{cs}(F)$ with $p(x) \leq q(T(x))$ for each $x \in E$. Compare with the definition in the case of normed spaces in 2, Definition 2.1], 3, p. 173] and 8, Definition 3.3.1]. Clearly every bounded below operator is injective. It is easy to see that an operator $T \in L(E, F)$ is bounded below if and only if $T$ is an isomorphism from $E$ into $F$, i.e. $T$ is a monomorphism in the sense of [11, vol. II, p. $2 \mathrm{ff}$.], i.e. $T$ is linear continuous injective and open into its image. The set of all monomorphisms from $E$ to $F$ is denoted by $m o(E, F)$.

An operator $T \in L(E, F)$ is called almost open, and we write $T \in a o(E, F)$ if for every $U \in \mathcal{U}_{0}(E)$ there is $V \in \mathcal{U}_{0}(F)$ such that $V \subseteq \overline{T(U)}$, the closure taken in $F$. Clearly every almost open operator has dense range. Almost open operators are precisely the nearly open operators with dense range in the sense of Pták (see [11, vol. II, p. 24]). The case of almost open operators between normed spaces is studied in [8, p. 65 ff.]. The class of almost open operators between Fréchet spaces coincides with the class of surjective operators by the Banach-Schauder open mapping theorem (see [11, vol. I, p. 166] or [15, Chapter 8]). The relation between 
the properties of $T \in L(E, F)$ mentioned above and its transpose $T^{t}: F^{\prime} \rightarrow E^{\prime}$ is analyzed in [3, 57.16-18], [8, 5.5.2-4], and [9, Section 2] for normed spaces $E$ and $F$. The situation for locally convex spaces is more complicated; we refer to Dierolf and Zarnadze [6].

\section{RESUlts}

Theorem 2.1. Assume that $m o(E, F)$ is not empty. The set $m o(E, F)$ is open in $L_{b}(E, F)$ if and only if $E$ is normable.

Proof. First we suppose that $m o(E, F)$ is open and non-empty. We select $T_{0} \in$ $m o(E, F)$, and a pair $B \in \mathcal{B}(E)$ and $V \in \mathcal{U}_{0}(F)$ such that every $S \in L(E, F)$ with $\left(S-T_{0}\right)(B) \subseteq V$ satisfies that $S \in m o(E, F)$. We claim that $V \cap T_{0}(E) \subseteq \overline{T_{0}(B)}$, which implies that $V \cap T_{0}(E)$ is a bounded neighbourhood of zero in $T_{0}(E)$, whence $E$ is normable by Kolmogorov's theorem. We prove the claim by contradiction: Suppose that there is $x \in E$ with $T_{0}(x) \in V \backslash \overline{T_{0}(B)}$, in particular $x \neq 0$. We apply the Hahn-Banach theorem to find $v \in F^{\prime}$ such that $v\left(T_{0}(x)\right)=1,\left|v\left(T_{0}(b)\right)\right|<1$ for all $b \in B$. We define $S: E \rightarrow F$ by $S(z):=T_{0}(z)-v\left(T_{0}(z)\right) T_{0}(x)$ for every $z \in E$. Clearly $S \in L(E, F)$. For every $b \in B$ we have $\left(T_{0}-S\right)(b)=v\left(T_{0}(b)\right) T_{0}(x) \in V$. By assumption $S \in m o(E, F)$. But $S(x)=0$, and this implies that $S$ is not injective, a contradiction.

Now we assume that $E$ is a normed space with a norm $\|$.$\| , and we fix T_{0} \in$ $m o(E, F)$. By definition, there is $q \in \operatorname{cs}(F)$ such that $\|x\| \leq q\left(T_{0}(x)\right)$ for each $x \in E$. If $S \in L(E, F)$ satisfies $\sup \left\{q\left(T_{0}(x)-S(x)\right) \mid\|x\| \leq 1\right\} \leq \frac{1}{2}$, we have

$$
\|x\| \leq q\left(T_{0}(x)\right) \leq q(S(x))+q\left(T_{0}(x)-S(x)\right) \leq q(S(x))+\frac{1}{2}\|x\| \quad \text { for } x \in E .
$$

This implies $\|x\| \leq 2 q(S(x))$ for each $x \in E$ and we conclude that $S \in m o(E, F)$.

Theorem 2.2. Assume that ao $(E, F)$ is not empty. The set ao $(E, F)$ is open in $L_{b}(E, F)$ if and only if the space $F$ is normable and for every $T \in$ ao $(E, F)$ there is $B \in \mathcal{B}(E)$ such that $\overline{T(B)}$ is a neighbourhood of the origin in $F$.

Proof. We suppose that $a o(E, F)$ is open in $L_{b}(E, F)$ and not empty. Let $T$ be an arbitrary element of $a o(E, F)$. By assumption there are $B \in \mathcal{B}(E)$ and $V \in \mathcal{U}_{0}(F)$ such that if $S \in L(E, F)$ satisfies $(S-T)(B) \subseteq V$, then $S \in a o(E, F)$. We claim that $V \subseteq \overline{T(B)}$, from where the necessity follows. Proceeding by contradiction, assume that there is $y \in V \backslash \overline{T(B)}$. By the Hahn-Banach theorem there is $v \in F^{\prime}$ with $v(y)=1,|v(T(b))|<1$ for every $b \in B$. We define $S \in L(E, F)$ by $S(z):=$ $T(z)-v(T(z)) y$. If $b \in B$, we get $(T-S)(b)=v(T(b)) y \in V$. On the other hand, $S(E) \subseteq \operatorname{ker} v$ since

$$
v(S(z))=v(T(z))-v(T(z)) v(y)=0, \quad z \in E .
$$

This implies that $S(E)$ is not dense in $F$, and $S$ cannot be almost open. A contradiction.

To prove the converse we fix $T \in a o(E, F)$. By assumption $F$ is normed and there is $B \in \mathcal{B}(E)$ such that $\overline{T(B)}$ contains the unit ball $U_{F}$ of $F$, so that the operator $\hat{T}:=T \mid E_{B}: E_{B} \rightarrow F$ is almost open between these two normed spaces. By [8] Theorem 3.4.3], there is $\epsilon>0$ such that if $\hat{R} \in L\left(E_{B}, F\right)$ and $\|\hat{R}-\hat{T}\|_{L\left(E_{B}, F\right)} \leq \epsilon$, then $\hat{R} \in a o\left(E_{B}, F\right)$. Now $\mathcal{W}\left(B, \epsilon U_{F}\right)$ is a neighbourhood of the origin in $L_{b}(E, F)$. 
If $S \in L(E, F)$ satisfies $(S-T) \in \mathcal{W}\left(B, \epsilon U_{F}\right)$, then $\hat{S}:=S \mid E_{B} \in L\left(E_{B}, F\right)$ satisfies $\|\hat{S}-\hat{T}\|_{L\left(E_{B}, F\right)} \leq \epsilon$. Therefore $\hat{S} \in a o\left(E_{B}, F\right)$ and this yields that $\overline{\hat{S}(B)}=\overline{S(B)}$ is a neighbourhood in $F$.

The following consequence follows from the Banach-Schauder open mapping theorem and it extends [2, Theorem 3.1].

Corollary 2.3. Let $E$ and $F$ be Fréchet spaces. Assume that there is a surjective operator from $E$ onto $F$. The set of all surjective operators from $E$ onto $F$ is open in $L_{b}(E, F)$ if and only if $F$ is a Banach space and for every surjective operator $T: E \rightarrow F$ there is a bounded subset $B$ of $E$ such that $T(B)$ is a neighbourhood in $F$.

Concerning the assumption in Theorems 2.1 and 2.2 and Corollary 2.3 it is worth mentioning that there are pairs of Banach spaces or Fréchet spaces $(E, F)$ such that $m o(E, F)=\emptyset$ and $a o(E, F)=\emptyset$. This is well known for $E=l_{p}$ and $F=l_{q}$ with $p \neq q$ (see [13, Chapter 2]). Vogt [19] and Bonet [4] investigated pairs of Fréchet spaces $(E, F)$ such that every $T \in L(E, F)$ maps a neighbourhood in $E$ into a bounded subset of $F$. These pairs satisfy that $m o(E, F)$ and $a o(E, F)$ are empty, if $E$ and $F$ are not normable.

Not every surjective operator from a Fréchet space $E$ onto a Banach space $F$ satisfies that the unit ball of the Banach space $F$ is contained inside the image of a bounded subset of $E$ : There is a Köthe echelon space $E=\lambda_{1}(A)$ which is a Montel space having a quotient isomorphic to $F=l_{1}$; see [11,31.5] or [15, 27.21 and 27.22]. The quotient map $q: E \rightarrow F$ does not lift bounded sets since the bounded subsets of $E$ are relatively compact.

The class of quasinormable spaces was introduced and studied by Grothendieck. It contains Banach and nuclear spaces, and it is also stable by quotients. Every $D F$-space in the sense of Grothendieck is quasinormable. A locally convex space is called quasinormable if for every $U \in \mathcal{U}_{0}(E)$ there is $V \in \mathcal{U}_{0}(E)$ such that for every $\epsilon>0$ there is $B \in \mathcal{B}(E)$ with $V \subseteq B+\epsilon U$. Quasinormable Fréchet spaces and their relevance for the lifting of bounded sets can be seen in [15, Chapter 26] and [17, Section 8.3]. Our next result extends Miñarro [14, Theorem 1]. We thank A. Peris for providing us with this elementary proof.

Proposition 2.4. (a) Let $E$ be a quasinormable space and let $F$ be a normed space. If $T \in a o(E, F)$, then there is $B \in \mathcal{B}(E)$ such that $U_{F} \subseteq \overline{T(B)}$.

(b) If $q$ is a surjective open operator between a locally complete quasinormable space $E$ and a Banach space $F$, then there is $B \in \mathcal{B}(E)$ such that $q(B)$ is a neighbourhood in $F$.

Proof. Part (b) is an easy consequence of (a) and the Banach-Schauder open mapping theorem, since $E_{B}$ is a Banach space.

We prove (a): Since $T$ is continuous we find $U \in \mathcal{U}_{0}(E)$ with $T(U) \subseteq \frac{1}{4} U_{F}$. As $E$ is quasinormable, we find $V \in \mathcal{U}_{0}(E)$ such that for all $\mu>0$ there is $B \in \mathcal{B}(E)$ with $\mu V \subseteq(B+U)$. We apply that $T$ is almost open to find $\lambda>0$ with $U_{F} \subset$ $\lambda \overline{T(V)} \subset \lambda T(V)+\frac{1}{4} U_{F}$. We select $C \in \mathcal{B}(E)$ with $\lambda V \subseteq C+U$ to conclude

$$
U_{F} \subseteq \lambda T(V)+\frac{1}{4} U_{F} \subseteq T(C)+T(U)+\frac{1}{4} U_{F} \subseteq T(C)+\frac{1}{2} U_{F} .
$$


Proceeding by recurrence we have, for each $n \in \mathbb{N}$,

$$
U_{F} \subseteq \sum_{k=0}^{n-1} 2^{-k} T(C)+\frac{1}{2^{n}} U_{F} \subseteq T(2 C)+\frac{1}{2^{n}} U_{F} .
$$

This yields $U_{F} \subseteq \overline{T(2 C)}$.

Corollary 2.5. (a) If $E$ is a quasinormable space and $F$ is a normed space, then $a o(E, F)$ is an open subset of $L_{b}(E, F)$.

(b) If $E$ is a locally complete quasinormable space and $F$ is a Banach space, then the set of surjective and open operators from $E$ onto $F$ is open in $L_{b}(E, F)$.

Corollary 2.5 extends the results of 8 , Theorem 3.4.3] and of [2, Theorem 3.1]. Simple examples in [2, p. 460] show that 2.5 b) does not hold for normed spaces. These ideas are exploited in our next result.

Theorem 2.6. (a) Every infinite-dimensional separable Banach space $X$ contains a dense subspace $E$ such that the set of surjective and open operators onto $E$ is not an open subset of the normed space $L(E)$.

(b) Every infinite-dimensional Banach space $X$ contains a proper dense subspace $F$ such that the set of surjective and open operators onto $F$ is an open subset of the normed space $L(F)$.

Proof. a) By a result of Ovsepian and Pełczyński [16], there are sequences $\left(x_{n}\right)_{n=1}^{\infty} \subseteq$ $X,\left\|x_{n}\right\|=1, \overline{\operatorname{span}\left\{x_{n} \mid n \in \mathbb{N}\right\}}=X$ and $\left(u_{n}\right)_{n=1}^{\infty} \subseteq X^{\prime}$ with $\left\|u_{n}\right\| \leq 2$ such that $u_{m}\left(x_{n}\right)=\delta_{n m}$ (Kronecker's delta). We set $E:=\operatorname{span}\left(x_{1}, x_{2}, \ldots\right)$. For $\epsilon>0$ we define $T_{\epsilon}: E \rightarrow E$ by

$$
T_{\epsilon}(x):=x-\epsilon \sum_{n=1}^{\infty} \frac{1}{2^{n+2}} u_{n}(x) x_{n+1} .
$$

The operator $T_{\epsilon}$ is clearly well-defined and continuous by the properties of the biorthogonal sequences $\left(x_{n}\right)_{n=1}^{\infty}$ and $\left(u_{n}\right)_{n=1}^{\infty}$. If $x \in E$ and $\|x\| \leq 1$, then

$$
\left\|\left(I-T_{\epsilon}\right)(x)\right\| \leq \frac{\epsilon}{2} \sum_{n=1}^{\infty} \frac{1}{2^{n+1}}\left\|u_{n}\right\|\left\|x_{n+1}\right\| \leq \epsilon .
$$

For each $x:=\sum_{i=1}^{k} \alpha_{i} x_{i} \in E$ with $\alpha_{k} \neq 0$, we have

$$
u_{k+1}\left(T_{\epsilon}(x)\right)=-\frac{\epsilon}{2} \frac{1}{2^{k+1}} \alpha_{k} \neq 0 .
$$

This implies that $x_{1} \notin T_{\epsilon}(E)$. The proof is complete in this case.

b) Let $X$ be an infinite-dimensional Banach space, and let $u: X \rightarrow \mathbb{K}$ be a non-continuous linear form. Then $F:=\operatorname{ker} u$ is a dense hyperplane of $X$. We first show the following observation.

Remark 2.7. Let $g \in L(X)$ be a surjective operator such that $g(F) \subseteq F$. Let $\tilde{g}:=g \mid F \in L(F)$ be the restriction of $g$ to $F$. Then:

(i) $g(X \backslash F) \subseteq X \backslash F$.

(ii) $\tilde{g}$ is surjective onto $F$.

(iii) $\operatorname{ker} g=\operatorname{ker} \tilde{g}$. 
Indeed, to show (i) suppose there is $z \in X \backslash F$ with $g(z) \in F$. Since $X=F \oplus \operatorname{span}(z)$, we conclude $g(X) \subseteq g(F) \oplus \operatorname{span}(g(z)) \subseteq F \neq X$, which is a contradiction, since $g$ is surjective in $X$. To show (ii), we pick $y \in F$. Since $g$ is surjective there is $z \in X$ with $g(z)=y \in F$. By (i) $z \in F$ and $\tilde{g}$ is surjective. Finally to prove (iii) we first observe that $\operatorname{ker} \tilde{g} \subseteq \operatorname{ker} g$. If $x \in \operatorname{ker} g \subseteq X$, we have $g(x)=0 \in F$. By (i) $x \in F$ and $g(x)=0$, i.e. $x \in \operatorname{ker} \tilde{g}$.

We continue with the proof of part (b). We fix $T \in L(F)$ which is surjective and open (i.e. a surjective homomorphism in the sense of [11, Chapter 32]). By density there is a unique extension $\hat{T} \in L(X)$ with $\hat{T} \mid F=T$. We apply [11, 32.5(3)] to conclude that $\hat{T}$ is a homomorphism in the Banach space $X$. Consequently $\hat{T}(X)$ is a closed subspace of $X$. Since $F \subseteq \hat{T}(X)$, we have that $\hat{T}$ is surjective. By [2] Theorem 3.1], there is $\epsilon>0$ such that if $R \in L(X)$ satisfies $\|R-\hat{T}\|_{L(X)} \leq \epsilon$, then $R$ is surjective. Let $S \in L(F)$ satisfy $\|S-T\|_{L(F)} \leq \epsilon$. The unique extension $\hat{S} \in L(X)$ of $S$ to $X$ satisfies $\|\hat{S}-\hat{T}\|_{L(X)} \leq \epsilon$, hence $\hat{S}$ is surjective onto $X$. We apply Remark 2.7 to $g:=\hat{S}, \tilde{g}=g \mid F=S$, to conclude that $S \in L(F)$ is surjective onto $F$, and $\operatorname{ker} \hat{S}=\operatorname{ker} S$. By [17] 2.6.18] $S$ is a homomorphism in $F$. Therefore $S \in L(F)$ is surjective and open.

In [2, Theorems 3.3 and 3.4] it is proved that, for Banach spaces $E$ and $F$, the set of isomorphisms from $E$ onto $F$ coincides with $m o(E, F) \cap \overline{a o(E, F)}$ and with $a o(E, F) \cap \overline{m o(E, F)}$, the closures taken in $L_{b}(E, F)$. Our examples below show that these results do not hold for Fréchet spaces $E, F$. We denote by $\omega$ the countable product of copies of the scalar field.

Example 2.8. The forward shift operator $F: \omega \rightarrow \omega$ defined as

$$
F\left(x_{1}, x_{2}, x_{3}, \ldots\right)=\left(0, x_{1}, x_{2}, x_{3}, \ldots\right)
$$

is a monomorphism which is not surjective. The operator $-F$ belongs to the closure of $a o(\omega, \omega)$. In fact $H_{n}:=-F+\frac{1}{n} I$ converges to $-F$ as $n$ tends to $\infty$ in $L_{b}(\omega, \omega)$, and $-F+\frac{1}{n} I$ is a surjective operator: indeed, for $y=\left(y_{j}\right)_{j=1}^{\infty} \in \omega$, we define $x=\left(x_{n}\right)_{n=1}^{\infty} \in \omega$ by $x_{j}:=n y_{j}+n^{2} y_{j-1}+\ldots+n^{j-1} y_{2}+n^{j} y_{1}$. Clearly $H_{n}(x)=y$. Consequently $\operatorname{mo}(\omega, \omega) \cap \overline{a o(\omega, \omega)}$ is not contained in the set of isomorphisms onto $\omega$.

Example 2.9. The backward shift operator $B: \omega \rightarrow \omega$ defined as

$$
B\left(x_{1}, x_{2}, x_{3}, \ldots\right)=\left(x_{2}, x_{3}, \ldots\right)
$$

is surjective and not injective, but it belongs to the closure of $m o(\omega, \omega)$ in $L_{b}(\omega, \omega)$. In fact, we define $H_{n}: \omega \rightarrow \omega, H_{n}\left(x_{1}, x_{2}, x_{3}, \ldots\right)=\left(x_{2}, x_{3}, \ldots, x_{n}, x_{1}, x_{n+1}, \ldots\right)$. Clearly $H_{n}(x)$ converges to $B(x)$ as $n$ tends to $\infty$ for each $x \in \omega$. By the BanachSteinhaus theorem [11 39.5(1)], $H_{n}$ tends to $B$ as $n$ tends to $\infty$ on every precompact subset of $\omega$. Since $\omega$ is a Montel space $H_{n}$ converges to $B$ as $n$ goes to $\infty$ in $L_{b}(\omega, \omega)$. Consequently $a o(\omega, \omega) \cap \overline{m o(\omega, \omega)}$ is not contained in the set of isomorphisms onto $\omega$.

More can be said about a sequence of operators which converges in a very strong sense to a surjective isomorphism $T \in L(E)$. We need the following extension of the classical result for Banach spaces which is due to Garnir, De Wilde and Schmets [7. p. 346]. Its proof uses the Neumann Series. An operator $T \in L(E, F)$ is called 
bounded (resp. compact) if there is $U \in \mathcal{U}_{0}(E)$ such that $T(U)$ is bounded (resp. relatively compact) in $F$.

Theorem 2.10 (Garnir, De Wilde, Schmets). Let $E$ be a complete locally convex space. Let $T \in L(E)$ be an operator such that there is $U \in \mathcal{U}_{0}(E)$ with $(I-T)(U)$ bounded in $E$ and $(I-T)(U) \subseteq \alpha U$ for some $0<\alpha<1$. Then $T$ is an isomorphism onto $E$, and $T^{-1}=\sum_{k=0}^{\infty}(I-T)^{k}$, the series converging in $L_{b}(E)$. If $(I-T)$ is compact no completeness assumption is needed on $E$.

Proposition 2.11. Let $E$ be a complete locally convex space, $T \in L(E)$ an isomorphism, and $\left(T_{n}\right)_{n=1}^{\infty}$ a sequence in $E$ such that there are $B \in \mathcal{B}(E), U \in \mathcal{U}_{0}(E)$ such that for all $\epsilon>0$ there is $n(\epsilon) \in \mathbb{N}$ with $\left(T-T_{n}\right)(U) \subseteq \epsilon B$ for all $n \geq n(\epsilon)$. Then there is $n_{0} \in \mathbb{N}$ such that $T_{n}$ is an isomorphism onto $E$ for all $n \geq n_{0}$.

Before we begin the proof we would like to note the following remark.

Remark 2.12. If $\left(T_{n}\right)_{n=1}^{\infty}$ and $T$ satisfy the assumption of the proposition, then $T_{n}$ converges to $T$ in the sense of Mackey in $L_{b}(E)$; in particular, $T_{n}$ converges to $T$ in $L_{b}(E)$ as $n$ tends to $\infty$.

Proof. Since $T$ is an isomorphism, $V:=T(U)$ is a neighbourhood in $E$ and for every $\epsilon>0$ there exists $n(\epsilon) \in \mathbb{N}$ such that for every $n \geq n(\epsilon)$ we have $\left(I-T_{n} T^{-1}\right)(V) \subseteq \epsilon B$. This implies that, for $n \geq n(1)$, the operator $\left(I-T_{n} T^{-1}\right)$ is bounded. There is $\epsilon_{0}>0$ such that $\epsilon_{0} B \subseteq \frac{1}{2} V$. Put $n_{0}:=n\left(\epsilon_{0}\right)$. If $n \geq n_{0}$, we have $\left(I-T_{n} T^{-1}\right)(V) \subseteq \frac{1}{2} V$. We can apply Theorem 2.10 to conclude that $T_{n} T^{-1}$ is an isomorphism onto $E$. This implies that $T_{n}$ is an isomorphism for $n \geq n_{0}$.

\section{REFERENCES}

1. M. Akkar, Sur le groupe des éléments inversibles d'une algèbre bornologique convexe. Qalgèbres bornologiques convexes, C. R. Acad. Sc. Paris 300 (1985) 35-38. MR 86e:46040

2. Y. A. Abramovich, C. D. Aliprantis and I. A. Polyrakis, Some remarks on surjective and bounded below operators, Atti Sem. Mat. Fis. Univ. Modena XLIV (1996) 455-464. MR 98b:47002

3. S. K. Berberian, Lectures in functional analysis and operator theory, Springer (1974). MR 54:5775

4. J. Bonet, On the identity $L(E, F)=L B(E, F)$ for pairs of locally convex spaces $E$ and $F$, Proc. Amer. Math. Soc. 99 (1987) 249-255. MR 88c:46009

5. P. G. Casazza and N. J. Kalton, Generalizing the Paley-Wiener perturbation theory for Banach spaces, Proc. Amer. Math. Soc. 127 (1999) 519-527. MR 99c:47008

6. S. Dierolf and D. N. Zarnadze, On homomorphisms between locally convex spaces, Note di Matematica XII (1992) 27-41. MR 95a:46003

7. H. G. Garnir, M. De Wilde and J. Schmets, Analyse fonctionnelle I, Birkhäuser Verlag, Basel und Stuttgart (1968). MR 40:6222

8. R. Harte, Invertibility and singularity for bounded linear operators, Marcel Dekker, New York and Basel (1988). MR 89d:47001

9. R. E. Harte, Almost open mappings between normed spaces, Proc. Amer. Math. Soc. 90 (1984) 243-249. MR 85b:47024

10. G. J. O. Jameson, Topology and normed spaces, Chapman and Hall, London (1974). MR 57:3828

11. G. Köthe, Topological vector spaces I and II, Springer Verlag, Berlin-Heidelberg-New York (1969) and (1979). MR 40:1750; MR 81g:46001

12. Kasahara, See Note p. 206, Bull. de la Soc. Math. France, Memory no. 31-3 (1972).

13. J. Lindenstrauss and L. Tzafiri, Classical Banach spaces I: Sequence spaces, Ergebnisse Math. 92 (1977). MR 58:17766

14. M. A. Miñarro, A characterization of quasinormable Köthe sequence spaces, Proc. Amer. Math. Soc. 123 (1995) 1207-1212. MR 95e:46006 
15. R. Meise and D. Vogt, Introduction to functional analysis, Clarendon Press, Oxford (1997). MR 98g:46001

16. R. I. Ovsepian and A. Pełczyński, On the existence of a fundamental total and bounded biorthogonal sequence in every separable Banach space, and related constructions of uniformly bounded orthonormal systems in $\mathcal{L}^{2}$, Studia Mathematica 54 (1975) 149-159. MR 52:14942

17. P. Pérez Carreras and J. Bonet, Barrelled locally convex spaces, North-Holland Math. Studies 131, Amsterdam (1987). MR 88j:46003

18. W. Rudin, Functional analysis, McGraw-Hill, New York (1973). MR 51:1315

19. D. Vogt, Frécheträume, zwischen denen jede stetige lineare Abbildung beschränkt ist, J. reine angew. Math. 345 (1983) 182-200. MR 85h:46007

Departamento de Matemática Aplicada, ets Arquitectura, Universidad Politécnica de Valencia, E-46071 Valencia, Spain

E-mail address: jbonet@mat.upv.es

Departamento de Matemática Aplicada, Fac. Informatica, Universidad Politécnica De Valencia, E-46071 Valencia, Spain

E-mail address: aconejero@mat.upv.es 